\title{
Clinical impact of strict criteria for selectivity and lateralization in adrenal vein sampling
}

\author{
Alessandro Gasparetto, John F. Angle, Pasha Darvishi, Colbey W. Freeman, \\ Ray G. Norby, Robert M. Carey
}

University of Virginia, USA

\begin{abstract}
INTRODUCTION: Selectivity index (SI) and lateralization index (LI) thresholds determine the adequacy of adrenal vein sampling (AVS) and the degree of lateralization. The purpose of this study was investigate the clinical outcome of patients whose adrenal vein sampling was interpreted using "strict criteria" (SC) (SI pre-stimuli $\geq 3, S I_{\text {post-stimuli }} \geq 5$ and $\left.L I_{\text {pre-stimuli }} \geq 4, L I_{\text {post-stimuli }} \geq 4\right)$. MATERIALS AND METHODS: A retrospective review of 73 consecutive AVS procedures was performed and 67 were technically successful. Forty-three patients showed lateralization and underwent surgery, while 24 did not lateralize and were managed conservatively. Systolic blood pressure (SBP), diastolic blood pressure (DBP), kalemia $\left(\mathrm{K}^{+}\right)$, and the change in number of blood pressure (BP) medications were recorded for each patient before and after AVS and potential surgery were performed. RESULTS: In the surgery group, BP and $\mathrm{K}^{+}$changed respectively from $160 \pm 5.3 / 100 \pm 2.0 \mathrm{mmHg}$ to $127 \pm 3.3 / 80 \pm 1.9(\mathrm{p}<0.001)$ and from $3.00 \pm 0.10$ to $4.4 \pm 0.09(\mathrm{p}<0.001)$. In the medically managed group, BP and $\mathrm{K}^{+}$changed respectively from $148 \pm 7.3 / 93 \pm 4.3$ to $135 \pm 3.3 / 86 \pm 1.9(p<0.001)$ and from $2.68 \pm 0.10$ to $4.3 \pm 0.09$. After surgery or AVS, the patients who took $\geq 3$ blood pressure medications were six $(14.0 \%)$ in the lateralized group and $22(91.7 \%)$ in the non-lateralized group $(p<0.001)$. CONCLUSIONS: AVS interpretation with SC leads to significant clinical improvement in both patients who underwent surgery and those managed conservatively.
\end{abstract}

Key words: Adrenal vein sampling, Primary aldosteronism, Selectivity index, Lateralization index

\section{INTRODUCTION}

Primary aldosteronism $(\mathrm{PA})$ is considered the most prevalent type of secondary hypertension, estimated to affect $5 \%$ to $14 \%$ of all hypertensive patients. ${ }^{1}$ Once the clinical diagnosis is confirmed by clinical and

Address for correspondence:

John F. Angle, 1215 Lee St Charlottesville, 22908, Virginia, USA;

Tel.: +1 434 9249401, E-mail: jfa3h@virginia.edu

Received: 23-02-2016, Accepted: 18-05-2016 biochemical screening, it is of paramount importance to determine whether the PA is the result of unilateral or bilateral aldosterone secretion.

Adrenal vein sampling (AVS) is a demanding procedure that consists of obtaining blood samples from the adrenal veins to measure concentrations of aldosterone and cortisol in order to differentiate unilateral from bilateral disease. ${ }^{2-4}$ For consistency of interpretation, the sample concentrations are translated 
into a selectivity index (SI), an expression of the accuracy of sampling, and a lateralization index (LI), an index of the lateralization of aldosterone secretion. The optimal values of SI and LI remain controversial: several case series have been published using different SI and LI values. ${ }^{3,5-6}$ The lack of established thresholds results in variable sensitivity and specificity of the AVS procedure..$^{7-9}$ The purpose of this study was to investigate the clinical outcome of patients whose adrenal vein sampling was interpreted using

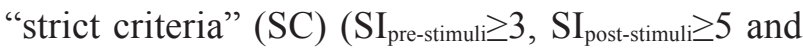
$\mathrm{LI}_{\text {pre-stimuli }} \geq 4, \mathrm{LI}_{\text {post-stimuli }} \geq 4$ ). The patients who did not lateralize using SC were divided into two subpopulations to determine whether lower LI was associated with a better clinical outcome compared to those with a LI closer to the SC.

\section{PATIENTS AND METHODS}

A retrospective review was conducted of 73 consecutive patients who underwent AVS between January 2004 and December 2014. All the patients were referred to a tertiary care academic institution. All the procedures were performed by a single experienced operator using a consistent technique. A Human Investigation Committee exemption was obtained. Data collected included the number of antihypertensive pharmacologic agents, serum $\mathrm{K}^{+}$levels, blood pressure measurements (BP), before and after each AVS was performed, and imaging.

\section{Study population}

The study population included treatment-resistant hypertensive patients with clinical and biochemical features of PA referred for AVS. Screening for PA had been performed in conformity with current guideline recommendations. ${ }^{3,10,11}$ As part of the screening process, hypokalemic patients had their serum $\mathrm{K}^{+}$ concentrations corrected with oral $\mathrm{K}^{+}$supplements and, when possible, antihypertensive medications that could interfere with the renin-angiotensin-aldosterone-system (RAAS; e.g. $\beta$-adrenergic antagonists, thiazide diuretics, amiloride, triamterene, angiotensin receptor blockers (ARBs) and angiotensin converting enzyme (ACE) inhibitors) were withdrawn within two weeks of testing. Slow release verapamil, hydralazine, doxazosin and/or methyldopa, agents which do not significantly influence the RAAS, were substituted when necessary to control BP. ${ }^{12}$ Spironolactone and eplerenone were withdrawn at least five weeks prior to testing. Plasma aldosterone concentration: plasma renin activity ratio (ARR) was measured in the morning with the patient in a seated position. The patients who presented with ARR $>30$ were subsequently scheduled for confirmatory testing with a saline suppression test. Correction of hypokalemia (serum $\mathrm{K}^{+}<3.5 \mathrm{mmol} / \mathrm{L}$ ) was provided before the test was performed. Plasma aldosterone concentration was measured at baseline and at 240 minutes after intravenous infusion of $2 \mathrm{~L}$ of $0.9 \%$ saline; failure of aldosterone to decrease to $<8 \mathrm{ng} / \mathrm{dL}$ with saline was considered to be diagnostic of PA. ${ }^{12}$ Patients with positive saline suppression test results and prior consent to undergo laparoscopic adrenalectomy in the event that a unilateral source of aldosterone hypersecretion was documented were scheduled for AVS. All the patients had at least one CT or MRI before undergoing AVS.

\section{AVS Procedure}

All AVS procedures were performed under intravenous conscious sedation. The intravenous sampling catheters were prepared with a side hole near the tip in order to improve blood sampling. ${ }^{2,5,13}$ Bilateral femoral venous access through appropriate introducer sheaths was obtained and catheters were advanced into the left and right adrenal veins. Inferior vena cava control samples were obtained through a 6-French left femoral introducer sheath. Once the position of the catheters was considered appropriate according to the operator's interpretation of adrenal venography, all the samples were collected simultaneously without further changes in catheter position. To achieve a higher selectivity, the left adrenal vein was catheterized with a microcatheter. Samples of right and left adrenal veins and infrarenal inferior vena cava (IVC) were performed simultaneously, although there is no evidence that this technique is superior to obtaining sequential samples. ${ }^{14}$ Each AVS consisted of at least four sets of samples: two sets of samples were collected 5 minutes apart before synthetic adrenocorticotropic hormone (ACTH) stimulation and two more were collected 20 and 40 minutes after $250 \mathrm{ug}$ of $\mathrm{ACTH}$ (cosyntropin; Amphastar, Rancho Cucamonga, California) had been administered.

Starting 2008, 31 patients underwent intraproce- 
dural cortisol measurements in an effort to reduce the number of non-diagnostic AVS procedures: the technique involved rapid assay of serum cortisol concentrations from each adrenal vein and the IVC on both the pre-stimulated samples (i.e. -40 and -20 minutes). Adrenal/peripheral cortisol ratios provided the operator with an intraprocedural feedback on when to reposition an adrenal vein catheter. In particular, when SI did not reach the threshold for selectivity for pre-stimuli samplings ( $\mathrm{SI} \geq 3$ ), the catheter was repositioned before obtaining the post-stimuli samplings.

The results of each AVS were interpreted by the same experienced endocrinologist. SI was calculated as $\left[\right.$ Cortisol $\left._{\text {adrenal vein }}\right] /\left[\right.$ Cortisol $\left._{\text {IVC }}\right]$ and LI as [Aldosterone/ Cortisol $]_{\text {adrenal vein }} /[\text { Aldosterone/Cortisol }]_{\text {contralateral adrenal }}$ vein. All AVS information was integrated with clinical and imaging data before the diagnosis of unilateral or bilateral PA was finalized. $\mathrm{SI}_{\text {pre-stimuli }} \geq 3, \mathrm{SI}_{\text {post-stimuli }}$ $\geq 5, \mathrm{LI}_{\text {pre-stimuli }} \geq 4$ and $\mathrm{LI}_{\text {post-stimuli }} \geq 4$ were used as the criteria for diagnosis of unilateral PA.

Two sets of non-stimulated and two sets of stimulated samples were routinely obtained. Each patient's diagnosis was based on the majority of selective set of samples suggesting the same diagnosis of unilateral or bilateral PA.

\section{Statistical Analysis}

Measurements are reported as mean $\pm \mathrm{SD}$. Normality of the distributions was confirmed by a ShapiroWilk test. Paired t-tests were used to compare pre-and post-treatment potassium and BP measurements in both the surgical and medical treatment groups. The unpaired t-test was used to compare the outcomes between the subpopulation whose LI were respectively above and below LI $I_{P C}$. A Chi-square test was used to compare the number of patients who changed BP medication class from $0-2$ to $\geq 3$ in the lateralized and non-lateralized groups and to compare them after surgery or AVS. Nodules dimension(s) and age were investigated as predictors of AVS outcome by univariate analysis. The software of Spotfire $\mathrm{S}$ plus version 8.5 (TIBCO INC., Palo Alto, CA) was used to conduct the data analyses.

\section{RESULTS}

Five of 73 patients were not included in the analysis due to loss of follow-up or lack of data. Nine (12.3\%) AVS procedures were non-diagnostic (not selective enough) after the first procedure and were successfully repeated. Sixty-seven patients had a diagnostic AVS after one or two procedures. Forty-three of 67 (64.2\%) patients demonstrated unilateral disease and underwent unilateral laparoscopic adrenalectomy. Twenty-four were diagnosed with bilateral aldosterone hypersecretion (Figure 1).

The mean age of the 43 patients that underwent surgery was $55.7 \pm 12.4$ years and $60.5 \%$ were male. The pathology reports of $36(83.7 \%)$ were consistent with aldosterone-producing adenomas adenoma (APA), one with carcinoma $(2.3 \%)$ and six with unilateral nodular adrenal hyperplasia (13.9\%). Four of the latter six patients showed full clinical improvement with normalization of serum potassium, normalization of the BP, and in three out of four, freedom from medication; these were ultimately considered as being unilateral hyperplastic adrenal glands. The remaining two showed only partial improvement of BP and $\mathrm{K}^{+}$levels and were successfully managed medically. Thirty-eight patients out of $43(88.4 \%)$ that underwent adrenalectomy showed improved control of potassium levels, while $37(86.0 \%)$ and $34(79.1 \%)$ respectively improved their BP control and reduced the amount of drugs to achieve it.

The mean systolic blood pressure (SBP) dropped from $160 \pm 5.3 \mathrm{mmHg}$ to $127 \pm 3.3$ ( $\mathrm{p}<0.001)$, as did the mean diastolic blood pressure (DBP) that fell from $100 \pm 2.0 \mathrm{mmHg}$ to $80 \pm 1.9(\mathrm{p}<0.001)$. Potassium levels went from $3.0 \pm 0.10$ to $4.4 \pm 0.09$ ( $p<0.001)$.

The mean age of the patients that were treated medically due to a non-lateralized AVS was $55.0 \pm 11.9$ years (58.3\% male). Mean BP fell from $148 \pm 7.3 / 93 \pm 4.3$ to $135 \pm 3.3 / 86 \pm 1.9(\mathrm{p}<0.001)$ and $\mathrm{K}^{+}$from $2.68 \pm 0.10$ to $4.3 \pm 0.09$ (Table 1 ).

Mean ARR at presentation and reduction in aldosterone post normal saline infusion in the lateralization group was $47.0 \pm 44.1$ and $14.2 \pm 29.2(\mathrm{ug} / \mathrm{dL})$, while in the non-lateralized it was $23.0 \pm 28.5$ and $8.7 \pm 17.7$ (ug/dL) ( $\mathrm{p}=0.042$ and 0.037$)$.

In the lateralization group, before surgery eight patients were taking $<3$ medications, while 35 were taking $\geq 3$; after treatment 37 patients reduced their 


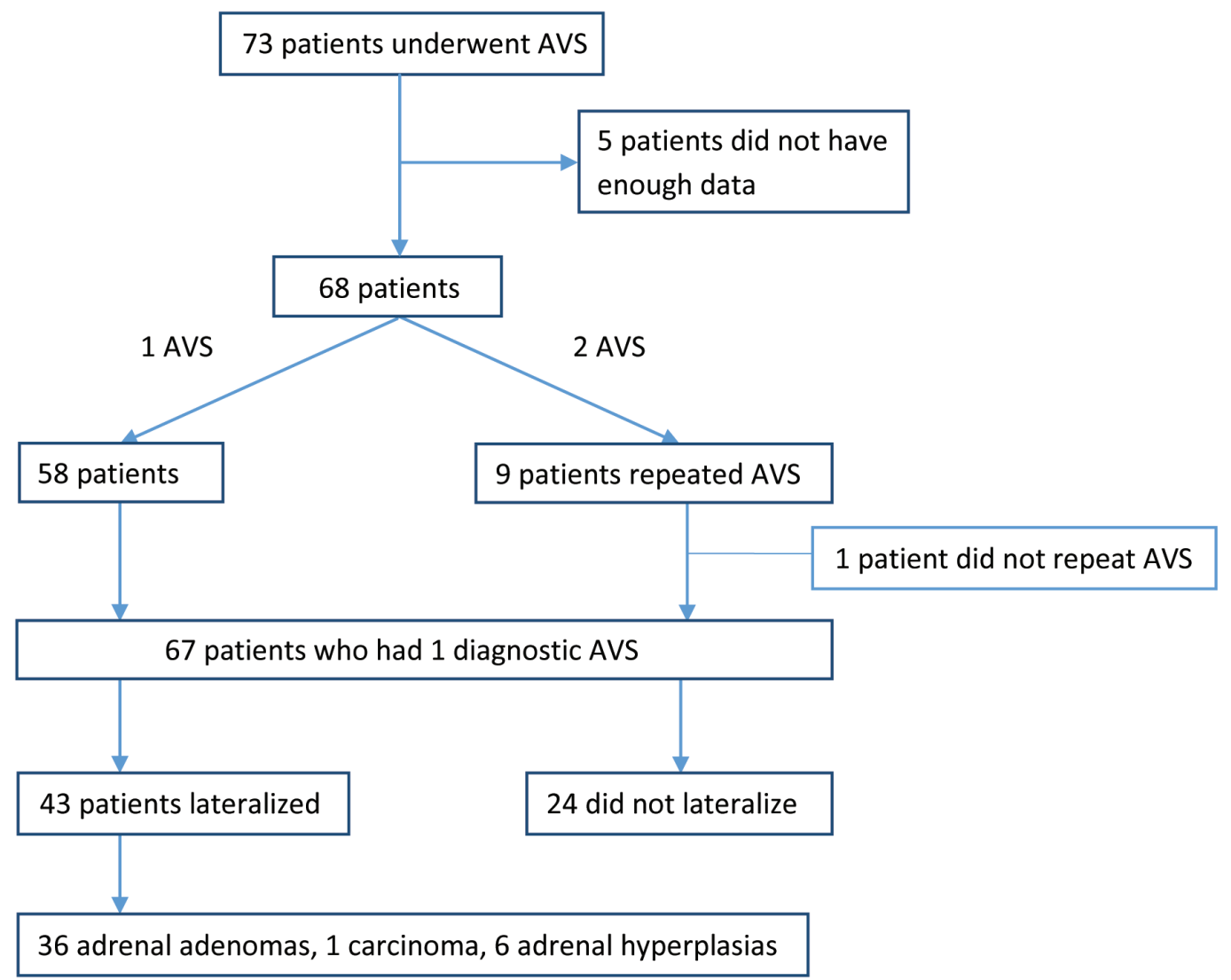

Figure 1. Study design.

medications to less than 3 and only six were still on 3 or more BP medications $(p<0.001)$ (Figure $2 a)$. In the non-lateralized group five patients were taking $<3$ medications, while 19 were taking $\geq 3$; after AVS the number of patients that took $\geq 3$ medications increased to $22(p=0.416)$ (Figure $2 b)$. The amount of BP medications at presentation was not statistically different between the patients who lateralized versus those who did not lateralize $(\mathrm{p}=0.532)$. After surgery or AVS the patients who took $\geq 3$ blood pressure medications was six (14.0\%) in the surgery group, while it was $22(91.7 \%)$ in the medically managed group $(\mathrm{p}<0.001)$.

Twenty out of 24 (83.3\%) patients who were managed with antihypertensive showed improved level of potassium and 20 improved their BP control after adjustment of the therapy.

Of the 24 patients who did not lateralize in $10 \mathrm{LI}$ was $\geq 2$, while in $14 \mathrm{LI}$ was $<2$. Intergroup comparison of the outcomes revealed no difference (Table 2).
Sixty-two patients had a CT, five an MR. Fifty-one patients displayed unilateral nodules, 44 were $>1 \mathrm{~cm}$, seven were $<1 \mathrm{~cm}$, eight showed no nodule or fullness, eight had bilateral nodules. Thirty-five $(79.5 \%)$ of the 44 patients that displayed a unilateral nodule $\geq 1 \mathrm{~cm}$ lateralized on the same side on AVS (Table 3 ).

\section{DISCUSSION}

AVS lacks a standard of reference for appropriate SI and LI and a gold standard for diagnosis in patients who do not undergo surgical resection. In addition, the pathology observed at surgery does not correlate uniformly with postoperative clinical outcomes. Letavernier et $\mathrm{a}^{15}$ demonstrated that, regardless of the pathologic findings of the adrenal glands, patients showing elevated lateralization indexes (LI $\geq 5$ ) are likely to improve clinically after unilateral adrenalectomy. This provides support for the assumption that the AVS diagnosis was correct in the six patients in this study with hyperplastic adrenal glands removed 
Table 1. Population characteristics and outcomes divided by AVS diagnosis. Mean follow up 16 months

\begin{tabular}{lc}
\hline Variable & $\begin{array}{c}\text { Outcomes } \\
\text { (mean } \pm \text { SD) }\end{array}$ \\
\hline 43 Patients who lateralized and underwent & \\
surgery & \\
Age (years) & $56 \pm 12$ \\
M:F (\%) & $61: 39$ \\
Systolic Blood Pressure (mmHg)* & \\
$\quad$ Pre-procedure & $160 \pm 5.3$ \\
$\quad$ Post-procedure & $127 \pm 3.3$ \\
Diastolic Blood Pressure (mmHg)* & \\
$\quad$ Pre-procedure & $100 \pm 2.0$ \\
$\quad$ Post-procedure & $80 \pm 1.9$ \\
Potassium (mmol/L)* & \\
Pre-procedure & $3.00 \pm 0.10$ \\
Post-procedure & $4.4 \pm 0.09$ \\
24 Patients who did not lateralize and were & \\
managed conservatively & \\
Age (years) & \\
M:F (\%) & \\
Systolic Blood Pressure (mmHg)* & \\
Pre-procedure & \\
$\quad$ Post-procedure & \\
Diastolic Blood Pressure (mmHg)* & \\
$\quad$ Pre-procedure & \\
Post-procedure & \\
Potassium (mmol/L)* & \\
Pre-procedure & \\
Post-procedure & \\
\hline & \\
\hline
\end{tabular}

$* \mathrm{P}<0.001$ Paired t-test comparison to the pretreatment value

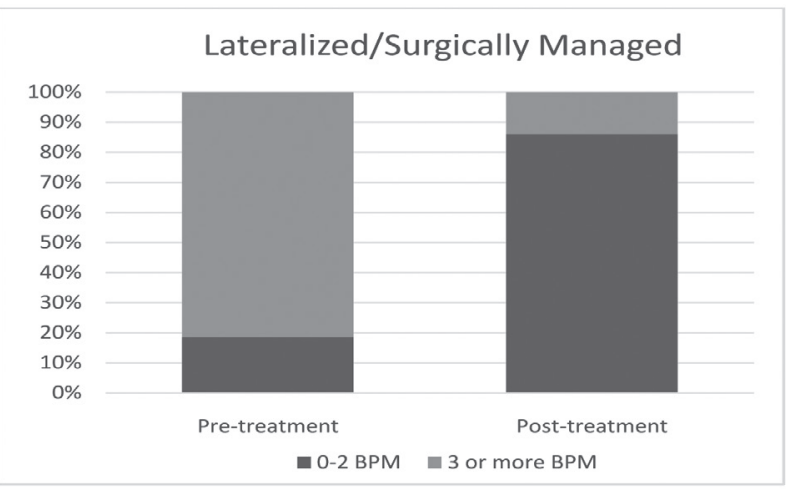

Figure 2a. Percentage of patients that switched from 3 blood pressure medications before surrenectomy to less than 3 after surrenectomy. BPM=blood pressure medications

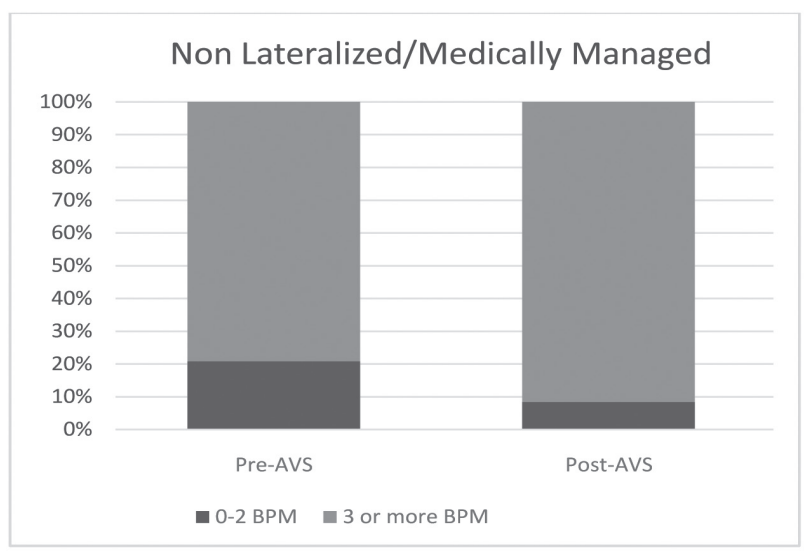

Figure 2b. Percentage of patients that changed from 3 blood pressure medications before AVS to less than 3 after AVS.

Table 2. Post-procedural outcomes comparison of 24 patients that were treated conservatively. The population was split in 10 and 14 patients whose SI and LI were respectively above and below the PC

\begin{tabular}{lccc}
\hline Variable & $\mathbf{L I}_{\mathbf{P C}}>\mathbf{L I}>\mathbf{L I}_{\mathbf{S C}} \mathbf{( 1 0 )}$ & $\mathbf{L I}_{\mathbf{P C}}>\mathbf{L I}_{\mathbf{S C}} \mathbf{( 1 4 )}$ & $\mathbf{p}$ \\
\hline $\mathrm{SBP}(\mathrm{mmHg})$ & $134 \pm 2.39$ & $134 \pm 4.07$ & $>0.999$ \\
$\mathrm{DBP}(\mathrm{mmHg})$ & $87 \pm 2.54$ & $86 \pm 2.23$ & $>0.999$ \\
$\mathrm{~K}^{+}(\mathrm{mmol} / \mathrm{L})$ & $2.90 \pm 0.12$ & $3.00 \pm 0.22$ & 0.207 \\
\hline
\end{tabular}

Table 3. Concordance of the imaging results with AVS

\begin{tabular}{lcccc}
\hline Imaging results & \multicolumn{3}{c}{ AVS result } & \\
\cline { 2 - 4 } & $\begin{array}{c}\text { Same side } \\
\text { LAT }\end{array}$ & $\begin{array}{c}\text { Contralateral } \\
\text { side }\end{array}$ & $\begin{array}{c}\text { Non } \\
\text { LAT }\end{array}$ & TOT \\
\hline Unilateral $>1 \mathrm{~cm}$ & 35 & 1 & 8 & 44 \\
Unilateral $<1 \mathrm{~cm}$ & 4 & 1 & 2 & 7 \\
Bilateral & 2 & N/A & 6 & 8 \\
Normal/fullness & 2 & N/A & 6 & 8 \\
& 43 & 2 & 22 & 67 \\
\hline
\end{tabular}

without an adenoma at pathology since the only available outcome considered was improvement or cure of hypertension. As surgery was demonstrated to be of little or no value in patients with bilateral adrenal disease, ${ }^{16}$ this study confirmed that applying SC provides significant clinical benefits both for the patients diagnosed with unilateral aldosteronism with complete healing and freedom from medications in $60.4 \%$ and for those with bilateral hyperaldosteronism who were able to receive a more targeted therapy without increasing the number of medications. 
Applying SC means inevitably that the specificity for correct cannulation and lateralization would drastically increase if compared to more permissive criteria of interpretation. ${ }^{9,7,17}$ The risk in this case is to misdiagnose unilateral adenomas as a bilateral hypertrophy. To address this issue, an additional comparison between the clinical outcome of the patients that would have lateralized using more permissive criteria PC (SI pre-stimuli $\geq 1.1 \mathrm{SI}_{\text {post-stimuli }} \geq 2$ and $\mathrm{LI}_{\text {pre-stimuli }} \geq 2 \mathrm{LI}_{\text {post-stimuli }} \geq 2$ ) and those who would not was run, which revealed no difference in outcome, meaning that a targeted drug therapy can provide an optimal increase of BP therapy and $\mathrm{K}^{+}$levels similarly in patients with $\mathrm{LI} \geq 2$ and in those with $\mathrm{LI}<2$ (Table 2). Furthermore, two discarded AVS that did not reach a high enough level of selectivity using SC would have shown non-lateralization if interpreted with $\mathrm{PC}$, but upon repeating the procedure, lateralization was confirmed by the AVS and subsequently by the pathology confirming an adenoma. These findings are consistent with multiple studies that pointed out the unreliability of SI and LI below the recommended criteria $(\mathrm{RC})\left(\mathrm{SI}_{\text {pre-stimuli }} \geq 2, \mathrm{SI}_{\text {post-stimuli }} \geq 3\right.$ and $\mathrm{LI}_{\text {pre-stimuli }} \geq 2$, $\left.\mathrm{LI}_{\text {post-stimuli }} \geq 4\right)^{5}{ }^{5,9,18-19}$

Given the distribution of the data with just one AVS falling in the "gray area" with LI and SI between the RC and $\mathrm{SC}$, it is possible that one or, more likely, a multitude of factors influenced such a wide diagnostic overlapping that brought the benefit of drastically reducing the doubts at the time of interpretation of the AVS. The purpose of using SC was to attain reasonable consistency and possibly outrank the performance of other criteria with lower LI and SI thresholds. In the attempt to reach this goal, the whole AVS technique was built with the awareness that these results would be achieved only by using multiple precautions. For example, duplicating preand post-stimuli samplings and trusting the AVS only if more than half of the single samples were diagnostic prevented us from repeating up to 9 and trusting up to 5 misdiagnosed AVS that would have pointed towards the opposite diagnosis if just one sample per AVS had been drawn. Similarly, a single experienced operator, withdrawal of the samplings before and after the administration of cosyntropin, the intra-procedural cortisol to obtain a feedback of proper positioning, the ready availability of 3D imaging in our opinion contributed to making a clear difference between AVS that lateralized and those that did not. These findings appear to be in contrast to other published series ${ }^{7,9,18}$ that showed a significant progressive lowering of the selective samples and an increase in the true positive lateralization upon raising the LI and SI thresholds.

It has long been debated whether imaging methods can recognize adrenal secreting nodules. ${ }^{4,20} \mathrm{An}$ additional analysis was performed to investigate if nodule dimension and the patient's age could predict the AVS outcome; in particular, features like the dimension of the nodule $\geq 1 \mathrm{~cm},{ }^{21}$ patients younger than 40 years old. ${ }^{13}$ Imaging characterization in this study failed to prove lateralization in 10 patients who had a unilateral nodule, and in eight who had a unilateral nodule $>1 \mathrm{~cm}$. Moreover, in two cases, with a unilateral $1 \mathrm{~cm}$ and $5 \mathrm{~mm}$ nodule, the AVS lateralized on the contralateral side. The first case was interpreted as a possible mistake in labelling the samples and upon repeating the procedure, AVS confirmed lateralization on the contralateral side compared to what the imaging showed. All 12 patients, after surgery, had pathology confirmation of adenoma. Eleven patients were younger than 40 years old, four had a unilateral nodule $\geq 1 \mathrm{~cm}$, but only $50 \%$ showed lateralization on AVS. These findings support the concerns raised by some author ${ }^{22,23}$ suggesting that mere imaging based lateralization must still be avoided.

The study had some limitations including the retrospective design and the loss to follow-up of some patients in the early phase.

\section{CONCLUSIONS}

SC of interpretation of AVS provided a significant clinical improvement in both the patients who underwent surgery and those who were managed medically. All the patients with SI and LI below the SC showed a significant clinical improvement with medical therapy.

\section{CONFLICT OF INTEREST}

The authors do not have any conflict of interest. 


\section{REFERENCES}

1. Gordon RD, Stowasser M, Tunny TJ, Klemm SA, Rutherford JC, 1994 High incidence of primary aldosteronism in 199 patients referred with hypertension. Clin Exp Pharmacol Physiol 21: 315-318.

2. Kline GA, Harvey A, Jones C, et al, 2008 Adrenal vein sampling may not be a gold-standard diagnostic test in primary aldosteronism: final diagnosis depends upon which interpretation rule is used. Variable interpretation of adrenal vein sampling. Int Urol Nephrol 40: 1035-1043.

3. Funder J, Carey R, Fardella C, et al, 2008 Case detection, diagnosis, and treatment of patients with primary aldosteronism: an Endocrine Society clinical practice guideline. J Clin Endocrinol Metab 93: 3266-3281.

4. Kempers MJ, Lenders JW, van Outheusden L, et al, 2009 Systematic review: diagnostic procedures to differentiate unilateral from bilateral adrenal abnormality in primary aldosteronism. Ann Intern Med 151: 329337.

5. Rossi, GP, Auchus RJ, Brown M, et al, 2014 An expert consensus statement on use of adrenal vein sampling for the subtyping of primary aldosteronism. Hypertension 63: 151-160.

6. Rossi GP, Barisa M, Allolio B, et al, 2012 The Adrenal Vein Sampling International Study (AVIS) for identifying the major subtypes of primary aldosteronism. J Clin Endocrinol Metab 97: 1606-1614.

7. Rossi GP, Pitter G, Bernante P, Motta R, Feltrin G, Miotto D, 2008 Adrenal vein sampling for primary aldosteronism: the assessment of selectivity and lateralization of aldosterone excess baseline and after adrenocorticotropic hormone (ACTH) stimulation. J Hypertens 26: 989-997.

8. Seccia TM, Miotto D, De Toni R, et al, 2009 Adrenocorticotropic hormone stimulation during adrenal vein sampling for identifying surgically curable subtypes of primary aldosteronism: comparison of 3 different protocols. Hypertension 53: 761-766.

9. Harvey A, Kline GA, Pasieka JL, 2006 Adrenal venous sampling in primary aldosteronism: comparison of radiographic with biochemical success and clinical decision making with 'less than ideal' results. Surgery 140: 847-853.

10. Nishikawa T, Omura M, Satoh F, et al, 2011 Guidelines for the diagnosis and treatment of primary aldosteronism - The Japan Endocrine Society 2009. Endocr J 58: 711-721.

11. Rossi GP, Auchus RJ, Brown M, et al, 2014 An expert consensus statement on use of adrenal vein sampling for the subtyping of primary aldosteronism. Hypertension 63: $151-160$.

12. Carey RM, 2012 Primary aldosteronism. J Surg Oncol 106: 575-579.

13. Young WF, Stanson AW, 2009 What are the keys to successful adrenal venous sampling (AVS) in patients with primary aldosteronism? Clin Endocrinol 70: 1417.

14. Carr CE, Cope C, Cohen DL, Fraker DL, Trerotola SO, 2004 Comparison of sequential versus simultaneous methods of adrenal venous sampling. See comment in PubMed Commons below. J Vasc Interv Radiol 15: 1245-1250.

15. Letavernier E, Peyrard S, Amar L, et al, 2008 Blood pressure outcome of adrenalectomy in patients with primary hyperaldosteronism with or without unilateral adenoma. J Hypertens 26: 1816-1823.

16. Sukor N, Gordon RD, Ku YK, Jones M, Stowasser M, 2009 Role of unilateral adrenalectomy in bilateral primary aldosteronism: a 22-year single center experience. J Clin Endocrinol Metab 94: 2437-2445.

17. Mulatero P, Bertello C, Sukor N, et al, 2010 Impact of different diagnostic criteria during adrenal vein sampling on reproducibility of subtype diagnosis in patients with primary aldosteronism. Hypertension 55: 667-673.

18. Lethielleux G, Amar L, Raynaud A, Plouin PF, Steichen O, 2015 Influence of diagnostic criteria on the interpretation of adrenal vein sampling. Hypertension 65: 849-854.

19. Rossi GP, Bernini G, Caliumi C, et al, 2006 A prospective study of the prevalence of primary aldosteronism in 1,125 hypertensive patients. J Am Coll Cardiol 48: 2293-2300.

20. Auchus RJ, Michaelis C, Wians JFH, et al, 2009 Rapid cortisol assays improve the success rate of adrenal vein sampling for primary aldosteronism. Ann Surg 249: 318-321.

21. Lau JHG, Sze WCC, Reznek RH, et al, 2012 A prospective evaluation of postural stimulation testing, computed tomography and adrenal vein sampling in the differential diagnosis of primary aldosteronism. Clin Endocrinol 76: 182-188.

22. Rosenquist KJ, Dluhy RG, 2011 Adrenal gland: uncertainty in the selective use of adrenal vein sampling. Nat Rev Endocrinol 7: 442-443

23. Sarlon-Bartoli G, Michel N, Taieb D, et al, 2011 Adrenal venous sampling is crucial before an adrenalectomy whatever the adrenal-nodule size on computed tomography. J Hypertens 29: 1196-1202. 